\title{
Evaluation of Novel Biomarkers for Early Diagnosis of Acute Kidney Injury in Asphyxiated Full-Term Newborns: A Case-Control Study
}

\author{
Ying Zhang ${ }^{a}$ Bili Zhang ${ }^{b}$ Dan Wang $^{a}$ Wujuan Shi ${ }^{a} \quad$ Anjie Zheng ${ }^{a}$ \\ a Department of Neonatology, Tianjin Children's Hospital, Tianjin, China; ${ }^{b}$ Department of Nephrology, Tianjin \\ Children's Hospital, Tianjin, China
}

\section{Significance of the Study}

- Previous studies have focused on urinary parameters, marker proteins or renal indices, but a holistic study combining all three has been rarely embarked upon.

- We explored the individual and combined ability of serum cystatin C, beta 2-microglobulin, urinary neutrophil gelatinase-associated lipocalin, and alpha 1-microglobulin to predict acute kidney injury in asphyxiated neonates.

- This study provides a reference for combined detection of renal injury after neonatal asphyxia.

\section{Keywords}

Acute kidney injury · Asphyxia · Full-term neonates · Urinary neutrophil gelatinase-associated lipocalin

\begin{abstract}
Objectives: To investigate the changes of serum cystatin $C$ (Cys-C), beta 2-microglobulin ( $\beta 2-M G)$, urinary neutrophil gelatinase-associated lipocalin (NGAL), and alpha 1-microglobulin (a1-MG) in asphyxiated neonates, and to evaluate the value of combined detection of multiple biomarkers in the early diagnosis of acute kidney injury (AKI) in asphyxiated neonates. Methods: A total of 110 full-term asphyxiated and 30 healthy neonates were included. The asphyxia neonates were divided into $A K I$ and non-AKI groups. Serum CysC, $\beta 2-M G$, urine NGAL, and a1-MG were measured $24 \mathrm{~h}$ after
\end{abstract}

birth. The diagnostic value of the biomarkers was determined using receiver operating characteristic (ROC) curves. Results: There was no significant difference in serum creatinine and blood urea nitrogen among the control group, moderate asphyxia group, and severe asphyxia group at 24 $\mathrm{h}$ after birth. Significant differences were noticed in terms of serum Cys-C, $\beta 2-M G$, urinary NGAL, and $\alpha 1-M G$ among the 3 groups. Moreover, with the aggravation of asphyxia, the above indicators gradually increased. There were significant differences in the 4 indicators between the AKI and non-AKI groups $(p<0.05)$. The area under the ROC curve of the above indicators was $0.670,0.689,0.865$, and 0.617 , respectively ( $p$ $<0.05)$. The sensitivity and specificity of the combined diagnosis of asphyxia neonatorum AKI with the 4 indicators were 0.974 and 0.506, respectively. Conclusions: Serum Cys-C, $\beta 2-$ MG, urine NGAL, and a1-MG are early specific indicators for

\begin{tabular}{|c|c|}
\hline KARGER & $\begin{array}{l}\text { (c) } 2019 \text { The Author(s) } \\
\text { Published by S. Karger AG, Basel }\end{array}$ \\
\hline $\begin{array}{l}\text { karger@karger.com } \\
\text { www.karger.com/mpp }\end{array}$ & $\begin{array}{l}\text { This is an Open Access article licensed under the Creative Commons } \\
\text { Attribution-NonCommercial- } 4.0 \text { International License (CC BY-NC) } \\
\text { (http://www.karger.com/Services/OpenAccessLicense), applicable to } \\
\text { the online version of the article only. Usage and distribution for com- } \\
\text { mercial purposes requires written permission. }\end{array}$ \\
\hline
\end{tabular}


the diagnosis of renal injury after neonatal asphyxia. Combined detection of these parameters could aid clinical evaluation of renal injury in asphyxiated neonates.

(C) 2019 The Author(s)

Published by S. Karger AG, Basel

\section{Introduction}

Asphyxia is an important cause of morbidity and mortality among neonates. The incidence of asphyxia is estimated to be 10 per 1,000 live births and is influenced by the local availability of medical resources [1]. It can lead to multi-organ dysfunction and a redistribution of cardiac output to maintain cerebral, cardiac, and adrenal perfusion while potentially compromising renal, gastrointestinal, and skin perfusion [2]. Renal injury in asphyxiated preterm infants has a high incidence $(30-55 \%)$ and mortality rate $60-66 \%$ [3]. Renal impairment in perinatal asphyxia has been noted by several studies in the past [4, 5]. However, diagnosis of acute kidney injury (AKI) is difficult in neonates as many of the established clinical and biochemical parameters are unreliable in this age group [6]. The immediate and long-term outcome depends to a large extent on the early recognition and appropriate management of complications.

Most renal function markers commonly used in clinical practice assess glomerular function. These include creatinine $(\mathrm{Cr})$-based parameters such as serum creatinine (sCr), from which the glomerular filtration rate (GFR) or endogenous $\mathrm{Cr}$ clearance is calculated, as well as measures of GFR calculated from serum cystatin C (Cys-C) [7]. Human Cys- $\mathrm{C}$ is a low-molecular weight protein, belonging to the cystatin superfamily of protease inhibitors, which is produced at a constant rate in all nucleated cells. Cys-C is freely filtered through the glomerular membrane, then completely reabsorbed and degraded by the proximal tubule. Serum Cys-C is being promoted as a more accurate estimate of neonatal GFR [8]. Serum beta 2-microglobulin $(\beta 2-M G)$ is of special interest to us, because it has been associated with the risk of end-stage kidney disease and death [9]. It belongs to low-molecular weight proteins that are freely filtered via the glomerular membrane and catabolized in tubules [10]. Urinary concentration of $\beta 2$ MG is a sensitive index of renal tubular function in asphyxiated neonates [11]. AKI biomarkers in the urine have been shown to be predictive of AKI and mortality in children undergoing cardiopulmonary bypass [12] and in critically ill preterm infants [13]. Urinary neutrophil gelatinase-associated lipocalin (NGAL) has bacteriostatic properties and contributes to innate immunity [14]. At the early phase of ischemia-induced AKI there is a rapid upregulation of NGAL mRNA in the Henle loop and proximal tubules, causing an increase in the synthesis and excretion of NGAL into the urine. In normal conditions, serum NGAL is filtered by the glomerulus and rapidly reabsorbed by the proximal tubule [15]. There is evidence that it can serve as a useful marker in pediatric populations with high predictive efficiency [16]. A previous study has suggested that the urinary measurement of alpha 1-microglobulin ( $\alpha 1-\mathrm{MG})$ can be a useful method of screening populations in whom there is a risk of tubular proteinuria whatever the underlying cause [17].

The ideal marker for detecting AKI should be upregulated shortly after an injury and independent of the level of GFR [18]. sCr levels and changes in urine output are the most commonly applied measures of renal function; however, using $\mathrm{Cr}$ to monitor renal function and to diagnose AKI is not ideal for many reasons: (i) the Cr value in a neonate reflects maternal $\mathrm{Cr}$; (ii) $\mathrm{Cr}$ is a measure of function (not injury), and it is a late marker of an acute injury; (iii) $>50 \%$ of nephrons must be compromised before changes in the Cr level become evident, so it is a late marker of significant renal dysfunction; (iv) at lower GFR, serum Cr will overestimate renal function, owing to tubular secretion of $\mathrm{Cr}$; (v) Cr varies by muscle mass, hydration status, age, and gender; and (vi) bilirubin and medications can affect Cr measurement by the Jaffe method [19].

Previous studies have focused on urinary parameters, marker proteins, or renal indices but a holistic study combining all of these has been embarked upon. To investigate the utility of serum and urine AKI biomarkers in asphyxiated neonates, we evaluated 6 previously identified candidate serum and urinary biomarkers: $\mathrm{sCr}$, blood urea nitrogen (BUN), serum Cys-C, $\beta 2-M G$, urinary NGAL, and $\alpha 1-$ MG. We explored the individual and combined ability of these biomarkers to predict AKI in asphyxiated neonates.

\section{Materials and Methods}

\section{Patients}

A total of 110 asphyxiated full-term newborns admitted in our hospital, between September 2016 and August 2017, were enrolled in our study. Thirty cases of normal newborn infants born in the same period were enrolled as healthy controls. All infants underwent physical examination and laboratory examination.

Inclusion criteria were based on the diagnostic criteria for neonatal asphyxia established by the Neonatal Resuscitation Group of the Chinese Medical Association Perinatal Medicine Branch in 2016. These criteria included infants born at term with perinatal asphyxia as evidenced by 3 or more of the following: (a) cord blood 
Table 1. General clinical data of patients with asphyxia and healthy controls

\begin{tabular}{llllcc}
\hline & $\begin{array}{l}\text { Healthy controls } \\
(n=30)\end{array}$ & $\begin{array}{l}\text { Moderate asphyxia } \\
(n=85)\end{array}$ & $\begin{array}{l}\text { Severe asphyxia } \\
(n=25)\end{array}$ & $\mathrm{X}^{2} / F$ & $p$ value \\
\hline Male/female, $n$ & $16 / 14$ & $58 / 27$ & $16 / 9$ & 2.146 & 0.342 \\
Gestational age, weeks & $39.09 \pm 1.21$ & $39.30 \pm 1.23$ & $39.30 \pm 1.52$ & 0.304 & 0.738 \\
Birth weight, g & $3,266 \pm 463.15$ & $3,319 \pm 456.84$ & $3,349 \pm 386.99$ & 0.255 & 0.775 \\
Birth length, cm & $49.17 \pm 1.82$ & $49.73 \pm 0.95$ & $49.55 \pm 1.32$ & 2.237 & 0.111 \\
\hline
\end{tabular}

Data are presented as means \pm SD unless otherwise indicated

$\mathrm{pH}<7.0$; (b) Apgar score $<6$ at $5 \mathrm{~min}$; (c) meconium-stained liquor; and $(\mathrm{d})$ changes in fetal heart rate [11].

Neonates were excluded if they had one or more of the following features: (a) prematurity or postmaturity; (b) neonates whose mothers had kidney disease, renal insufficiency, or who have received nephrotoxic drugs like aminoglycosides; (c) and neonates with significant illness (e.g., severe infection, jaundice, primary glomerular diseases such as nephritis), congenital malformations, or who were receiving drugs (e.g., aminoglycosides) which may affect the renal function.

Term normal appropriate for gestational age newborns delivered during the study period matched for hours after birth and sex were taken as controls from the postnatal ward of the same hospital. Gestational age of all the neonates was determined from the date of the last menstrual period of the mother and modified Ballard's scoring system.

The asphyxiated neonates were further grouped into moderate and severe asphyxia on the basis of Apgar score and umbilical cord $\mathrm{pH}$ : moderate asphyxia - Apgar score of 1 or $5 \mathrm{~min} \leq 7$ points and an umbilical cord $\mathrm{pH}<7.2$; severe asphyxia - Apgar score of $1 \mathrm{~min}$ $\leq 3$ points and/or Apgar score of $5 \mathrm{~min} \leq 5$ points and an umbilical cord $\mathrm{pH}<7.0$.

\section{Detection Index and Methods}

Venous blood (1-3 mL) was collected at $24 \mathrm{~h}$ of life for the estimation of blood Cys-C and $\beta 2-M G$. Urine samples $(10 \mathrm{~mL})$ were simultaneously collected using commercially available pediatric urine bags. Care was taken to prevent leakage and contamination of urine with stool. Specimens were preserved at low temperature after centrifugation (venous blood: 3,800 rpm for $8 \mathrm{~min}$; urine sample: $1,800 \mathrm{rpm}$ for $5 \mathrm{~min}$ ), and then sent to the laboratory. $\mathrm{sCr}$, Cys-C, $\beta 2-M G, B U N$, urinary NGAL, and $\alpha 1-M G$ were measured using an automatic biochemistry analyzer (Cobas 8000 Modular Analyzer Series C701; Roche, Mannheim, Germany).

The precision, accuracy, linearity, and anti-jamming performances were evaluated during the tests on a fully automated biochemical analyzer. sCr and BUN were measured by enzyme methods. A good accuracy was obtained for $\mathrm{sCr}$, with an intraspecific coefficient of variation $(\mathrm{CV})$ of $\leq 0.8 \%$ and an interspecific $\mathrm{CV}$ of $\leq 2.0 \%$. Good reproducibility of standard duplicates was obtained for BUN, with an average signal confidence of variability of $6.5 \%$. Blood Cys- $\mathrm{C}$ concentration was determined by latex-enhanced immunoturbidimetric assay with a detection interval between 0.40 and 6.80 $\mathrm{mg} / \mathrm{L}$. $\beta 2-\mathrm{MG}$ levels were measured using an end-point immunoturbidimetric assay, which employs a polyclonal latex-coated rabbit anti-human $32-$ MG antibody, on the Roche Modular P Analyzer. The assay has traceability to the 1st International Standard Preparation (1986, WHO) (Roche Tina-Quant Serum $\beta 2-$ MG Assay Kit, Cat. No. 11660551). The lowest tested concentration with a CV $\leq 20 \%$ was taken as the limit of quantification. The lower reporting limit was taken as the lowest linear concentration whose CV was $<20 \%$.

Urinary NGAL was measured by the Neutrophil GelatinaseAssociated Lipocalin Assay Kit by Latex using the enhanced immunoturbidimetry method. A good accuracy was obtained, with an intraspecific CV of $\leq 5 \%$ and an interspecific CV of $\leq 10 \%$. The measured values were linearly related to the theoretical values when the concentrations of NGAL were in the range of 25-3,000 $\mathrm{ng} / \mathrm{mL}(r \geq 0.990)$. a1-MG was measured by an immunoturbidimetric assay. A good accuracy was obtained, with an intraspecific $\mathrm{CV}$ of $\leq 10 \%$ and an interspecific CV of $\leq 10 \%$. The measured values were linearly related to the theoretical values when the concentrations of NGAL were in the range of $6-110 \mathrm{mg} / \mathrm{L}(r \geq 0.990)$.

The diagnostic criteria for AKI in asphyxiated neonates were as follows: reduction in kidney function within $48 \mathrm{~h}$ (currently defined as an absolute increase in $\mathrm{sCr}$ of $\geq 0.3 \mathrm{mg} / \mathrm{dL}[\geq 26.4 \mu \mathrm{mol} / \mathrm{L}]$ ), a percentage increase in $\mathrm{sCr}$ of $\geq 50 \%$ (1.5-fold from baseline), or a reduction in urine output (documented oliguria of $<0.5 \mathrm{~mL} / \mathrm{kg} / \mathrm{h}$ for more than $6 \mathrm{~h}$ ) [20].

\section{Statistical Analysis}

The normality of the data was evaluated by the KolmogorovSmirnov test. Continuous data with normal distribution were presented as means \pm SD and compared using the Student $t$ test. Variables with non-normal distribution were expressed as median (interquartile range) and compared using the Mann-Whitney test. Qualitative data were expressed as numbers or percentages and compared using the $\chi^{2}$ test. Statistical analysis was performed by SPSS 19.0 software (SPSS Inc., USA). Analysis of diagnostic susceptibility and specificity of $\beta 2-\mathrm{MG}$, Cys-C, $\alpha 1-\mathrm{MG}$, and NGAL were realized using the receiver operating characteristic curve (ROC) and the area under the curve (AUC). $p<0.05$ was considered statistically significant.

\section{Results}

\section{Baseline Characteristics}

A total of 110 asphyxiated full-term newborns (85 cases in the moderate asphyxia group and 25 cases in the severe asphyxia group) and 30 cases of normal newborn infants were enrolled in our study. The baseline characteristics of 
Table 2. Details of the AKI and Non-AKI groups

\begin{tabular}{|c|c|c|c|c|}
\hline & AKI $(n=37)$ & Non-AKI $(n=73)$ & $\mathrm{X}^{2} / F$ & $p$ value \\
\hline Male/female, $n$ & $29 / 8$ & $45 / 28$ & 3.123 & 0.077 \\
\hline Gestational age, weeks & $39.54 \pm 1.22$ & $39.18 \pm 1.32$ & -1.416 & 0.160 \\
\hline Birth weight, g & $3,322 \pm 408.76$ & $3,328 \pm 458.34$ & 0.076 & 0.939 \\
\hline Birth length, $\mathrm{cm}$ & $49.77 \pm 1.26$ & $49.84 \pm 0.83$ & -0.349 & 0.728 \\
\hline
\end{tabular}

Data are presented as means \pm SD unless otherwise indicated.

Table 3. Levels of biomarkers in asphyxia and healthy groups $24 \mathrm{~h}$ after birth

\begin{tabular}{lclllll}
\hline Groups & $\mathrm{sCr}, \mu \mathrm{mol} / \mathrm{L}$ & $\mathrm{BUN}, \mathrm{mmol} / \mathrm{L}$ & $\beta 2-\mathrm{MG}, \mathrm{mg} / \mathrm{L}$ & $\mathrm{Cys}-\mathrm{C}, \mathrm{mg} / \mathrm{L}$ & $\mathrm{NGAL}, \mu \mathrm{g} / \mathrm{L}$ & $\alpha 1-\mathrm{MG}, \mathrm{mg} / \mathrm{L}$ \\
\hline Healthy controls $(n=30)$ & $49.25 \pm 7.42$ & $3.35 \pm 1.16$ & $2.25 \pm 0.31$ & $1.00 \pm 0.20$ & $59 \pm 12$ & $8.95(4.25)$ \\
Moderate asphyxia $(n=85)$ & $51.03 \pm 9.44$ & $3.89 \pm 1.27$ & $2.89 \pm 0.84$ & $1.28 \pm 0.26$ & $108 \pm 31$ & $36.6(31.90)$ \\
Severe asphyxia $(n=25)$ & $52.74 \pm 8.58$ & $4.01 \pm 1.38$ & $3.54 \pm 0.98$ & $1.81 \pm 0.39$ & $268 \pm 57$ & $68.70(7.79)$ \\
\hline F/Z & 1.057 & 2.446 & 18.342 & 60.125 & 280.22 & 9.792 \\
\hline$p$ value & 0.350 & 0.090 & 0.000 & 0.000 & 0.000 & 0.000 \\
\hline
\end{tabular}

Data are presented as means \pm SD or median (IQR). sCr, serum creatinine; Cys-C, cystatin C; $\beta 2-\mathrm{MG}$, beta 2-microglobulin; NGAL, urinary neutrophil gelatinase-associated lipocalin; $\alpha 1-\mathrm{MG}$, alpha 1-microglobulin.

Table 4. Levels of biomarkers in the AKI and Non-AKI groups $24 \mathrm{~h}$ after birth

\begin{tabular}{lllcccc}
\hline Group & sCr, $\mu \mathrm{mol} / \mathrm{L}$ & $\mathrm{BUN}, \mathrm{mmol} / \mathrm{L}$ & $\beta 2-\mathrm{MG}, \mathrm{mg} / \mathrm{L}$ & $\mathrm{Cys}-\mathrm{C}, \mathrm{mg} / \mathrm{L}$ & $\mathrm{NGAL}, \mu \mathrm{g} / \mathrm{L}$ & $\alpha 1-\mathrm{MG}, \mathrm{mg} / \mathrm{L}$ \\
\hline AKI $(n=37)$ & $51.36 \pm 10.7$ & $4.35 \pm 1.03$ & $3.70 \pm 1.17$ & $1.96 \pm 0.34$ & $270 \pm 88$ & $53.3(68.6)$ \\
Non-AKI $(n=73)$ & $48.15 \pm 9.26$ & $4.06 \pm 1.14$ & $3.09 \pm 0.60$ & $1.78 \pm 0.28$ & $93 \pm 52$ & $38.20(27.90)$ \\
$t / Z$ & 1.625 & 1.301 & -3.588 & -2.985 & 13.247 & -2.347 \\
$p$ value & 0.107 & 0.196 & 0.001 & 0.004 & 0.000 & 0.019 \\
\hline
\end{tabular}

Data are presented as means \pm SD or median (IQR). sCr, serum creatinine; Cys-C, cystatin C; $\beta 2-\mathrm{MG}$ : beta 2-microglobulin; NGAL, neutrophil gelatinase-associated lipocalin; al-MG: alpha 1-microglobulin.

the 3 groups are shown in Table 1 . No significant differences were observed among the 3 groups in terms of gender, gestational age, birth weight, and birth length $(p>0.05)$.

Meanwhile, 110 asphyxiated full-term newborns were divided into the AKI group and the non-AKI group according to the diagnostic criteria for AKI in asphyxiated neonates [20]. The baseline characteristics in the AKI and non-AKI groups are shown in Table 2. Differences in gender, gestational age, birth weight, and birth length had no statistical significance $(p>0.05)$.

\section{Biomarker Differences in Asphyxia Infants and \\ Healthy Controls}

There was no significant difference in terms of $\mathrm{sCr}$ and BUN among the control group, moderate asphyxia group, and severe asphyxia group $24 \mathrm{~h}$ after birth $(p>$ $0.05)$, while significant differences were noticed in terms of serum Cys-C, $\beta 2-\mathrm{MG}$, urine NGAL, and $\alpha 1-\mathrm{MG}$ among the 3 groups $(p<0.05)$. The above indicators showed a gradual upward trend with the aggravation of asphyxia (Table 3).

Biomarker Differences in Infants with and without AKI

There were no significant differences in levels of $\mathrm{sCr}$ and BUN between the AKI and non-AKI groups, while the levels of Cys-C and $32-M G$ in blood, and NGAL and a1-MG in urine in the AKI group were significantly higher than those in the non-AKI group $(p<0.05$; Table 4). 


\section{ROC Analysis of Marker Proteins}

Using ROC analysis, we calculated the sensitivity and specificity for each marker's ability to detect AKI (Fig. 1). Values for NGAL gave the best diagnostic performance, with an AUC of 0.865. Analysis of Cys-C concentrations yielded an AUC of 0.670, and $\beta 2-M G$ and $\alpha 1-M G$ achieved a comparable AUC of 0.689 and 0.617 , respectively. Table 5 shows the results of the ROC analysis for all markers at $24 \mathrm{~h}$ after birth. The sensitivity and specificity of combined diagnosis of AKI in asphyxiated neonates with the above 4 indicators were 0.974 and 0.506 , respectively, indicating that the combined detection of multiple biomarkers could be helpful in the clinical evaluation of AKI in asphyxiated neonates.

\section{Discussion}

In this study, we evaluated the association of 6 serum and urine biomarkers with AKI in asphyxiated neonates. We found that the levels of Cys-C and $\beta 2-M G$ in blood, and NGAL and $\alpha 1-\mathrm{MG}$ in urine measured $24 \mathrm{~h}$ after birth were associated with AKI. These data suggest their potential as biomarkers of AKI.

Perinatal asphyxia is one of the most important causes of neonatal mortality and morbidity. AKI is independently associated with poor outcomes in the critically ill patient. The standard kidney function biomarker, sCr, shows a demonstrable rise in concentration many hours to days after insult to the kidney. Thus, Cr-based AKI diagnosis is likely delayed, rendering treatments to mitigate or prevent AKI ineffective. Neonatal AKI is further confounded by the fact that $\mathrm{sCr}$ concentrations in infants actually reflect maternal levels.

The diagnosis of AKI is usually based on changes in sCr, but such measurements are a poor marker of acute deterioration in kidney function and hence biomarkers are gaining importance. Biomarkers like serum Cys-C, urine interleukin-18 (IL-18), urine kidney injury molecule-1, urine NGAL, IL-18, glutathione-S-transferase-pi, and gamma-glutathione-S-transferase have been used in a few studies on AKI [21,22].

Estimation of urinary $\beta 2-\mathrm{MG}$ as an indicator of proximal renal tubular dysfunction in asphyxiated full-term newborns has been documented [23]. Cys-C is normally filtered freely and is completely reabsorbed and catabolized within the proximal tubule [24]. Urine Cys-C levels increase with structural and functional renal tubular impairment independent of GFR [25]. It is highly predictive of AKI in children and adults who undergo cardiopulmonary bypass surgery and kidney transplantation [26]. Stud-

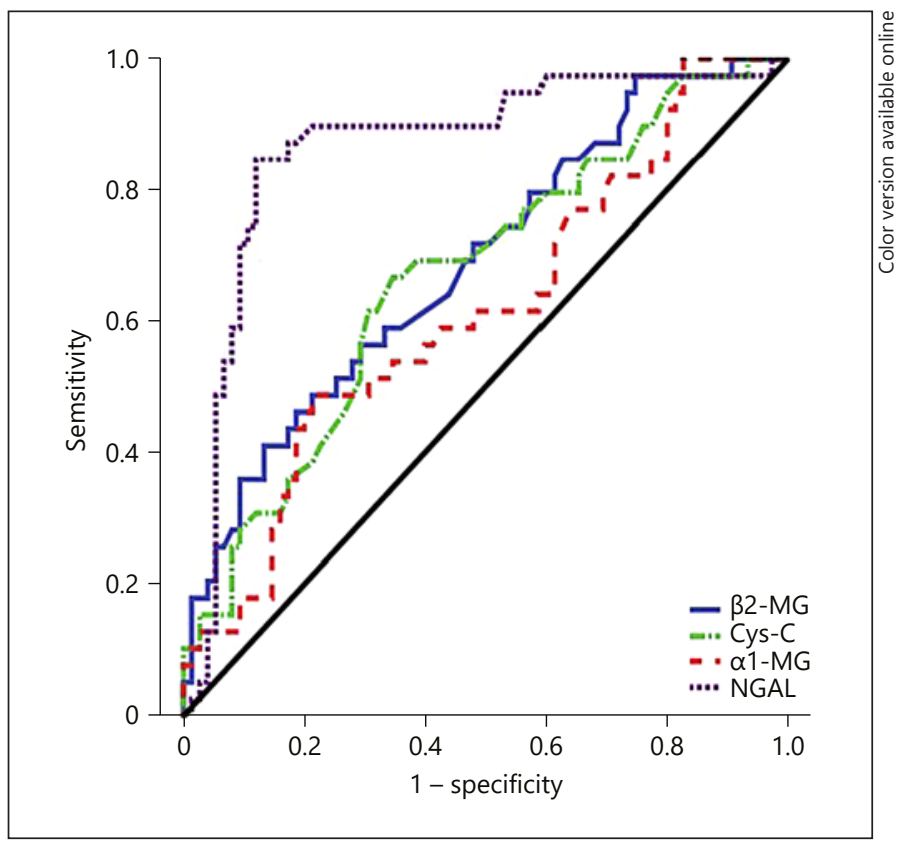

Fig. 1. ROC curves of blood Cys-C, $\beta 2-M G$, urinary NGAL, and a1-MG in diagnosing AKI after neonatal asphyxia. Cys-C, cystatin C; $\beta 2-M G$, beta 2-microglobulin; NGAL, neutrophil gelatinaseassociated lipocalin; a1-MG, alpha 1-microglobulin.

ies on 22 cases of children with burns admitted to hospital within $12 \mathrm{~h}$ revealed that there were no significant differences between the $\mathrm{sCr}$ level at 1-5 days in the AKI and the non-AKI groups [27]. By contrast, blood and urine NGAL levels increased considerably in the AKI group, which indicated the sensitivity of NGAL for early detection of AKI [27]. More recently, urinary NGAL was shown to be a good predictor of AKI in 108 term asphyxiated neonates with an AUC of 0.724 when a cutoff of $250 \mathrm{ng} / \mathrm{mL}$ was used $(56 \%$ AKI prevalence) [28]. Hoffman et al. [28] included 25 infants with hypoxic-ischemic encephalopathy receiving hypothermia in a study investigating a panel of urinary biomarkers for their ability to predict AKI in critically ill neonates. Previous studies have shown that urinary concentrations of some biomarkers are dependent on gestational age in children without AKI [29]. This might be secondary to the inability of immature tubules to reabsorb these proteins in underdeveloped kidneys. Controlling for this important confounder is necessary to ensure that the associations between urine biomarkers and AKI are not simply a reflection of tubular maturation [22].

This study has several limitations. First, the study is limited by the relatively small number of patients. Therefore, large-scale, head-to-head comparisons of the different biomarkers are required in the future. Another important lim- 
Table 5. Analysis of $\beta 2-\mathrm{MG}$, Cys-C, $\alpha 1-\mathrm{MG}$, and NGAL

\begin{tabular}{llllll}
\hline & $24-\mathrm{h}$ AUC & $95 \%$ CI & Sensitivity, \% & Specificity, \% & Cutoff \\
\hline 32-MG & 0.689 & $0.586-0.791$ & 0.487 & 0.787 & $3.49 \mathrm{mg} / \mathrm{L}$ \\
Cys-C & 0.670 & $0.565-0.774$ & 0.615 & 0.693 & $1.86 \mathrm{mg} / \mathrm{L}$ \\
al-MG & 0.617 & $0.508-0.726$ & 0.461 & 0.8 & $57.35 \mathrm{mg} / \mathrm{L}$ \\
NGAL & 0.865 & $0.788-0.943$ & 0.846 & 0.880 & $109.5 \mu \mathrm{g} / \mathrm{L}$ \\
\hline
\end{tabular}

Cys-C, cystatin C; $\beta 2-M G$, beta 2-microglobulin; NGAL, urinary neutrophil gelatinase-associated lipocalin; a1-MG, alpha 1-microglobulin.

itation of this study is the possibility that other confounders besides gestational age could explain the association between AKI and these biomarkers. Because of limitations in sample size, we were not able to control for other potential confounders, such as inflammatory changes, birth weight, maternal and infant genetic variations, and others. Our selection criteria led to a heterogeneous population of sick newborns. Studies with larger populations will control for these limitations. Third, newborns with moderate to severe encephalopathy underwent therapeutic hypothermia in our study, and this factor may affect the renal outcome. Lastly, further studies about the association of AKI with respect to gestational age and gender are needed.

\section{Conclusion}

Neonatal asphyxia can cause changes in glomerular and tubular function, and more severely asphyxiated neonates are more likely to experience renal failure compared to those with milder asphyxia. Serum Cys-C and $\beta 2-M G$, and urine NGAL and $\alpha 1-M G$ levels are early and specific indicators of renal damage after asphyxia. The combined detection of multiple biomarkers may contribute to clinical evaluation of renal injury in asphyxiated neonates. Because of limitations in sample size and the heterogeneous population of sick newborns, this study was not able to control for other potential confounders. Therefore, a large-scale, head-to-head comparison of serum and urinary levels is required to ascertain which of the two is the more useful test.

\section{Statement of Ethics}

This study was approved by the Ethics Committee of the local hospital and informed consent was obtained from guardians of all the participants.

\section{Disclosure Statement}

The authors declare that they have no conflict of interest.

\section{Funding Sources}

No funding was received for this study.

\section{References}

1 McGuire W. Perinatal asphyxia. BMJ Clin Evid. 2007 Nov;2007:379.

2 Durkan AM, Alexander RT. Acute kidney injury post neonatal asphyxia. J Pediatr. 2011 Feb;158(2 Suppl):e29-33.

3 Beck S, Wojdyla D, Say L, Betran AP, Merialdi M, Requejo JH, et al. The worldwide incidence of preterm birth: a systematic review of maternal mortality and morbidity. Bull World Health Organ. 2010 Jan;88(1):31-8.

4 Perlman JM, Tack ED. Renal injury in the asphyxiated newborn infant: relationship to neurologic outcome. J Pediatr. 1988 Nov; 113(5):875-9.

5 Nouri S, Mahdhaoui N, Beizig S, Zakhama R, Salem N, Ben Dhafer S, et al. [Acute renal fail- ure in full term neonates with perinatal asphyxia. Prospective study of 87 cases]. Arch Pediatr. 2008 Mar;15(3):229-35.

6 el-Mauhoub M, Kishan J, Jaiswal OP, Asbali AA, el houni N. Urinary beta 2-microglobulin as an indicator of proximal renal tubular dysfunction in asphyxiated full-term newborns. Pediatr Nephrol. 1994 Dec;8(6):787.

7 Heise D, Waeschle RM, Schlobohm J, Wessels J, Quintel M. Utility of cystatin C for assessment of renal function after cardiac surgery. Nephron Clin Pract. 2009;112(2):c107-14.

8 Kandasamy Y, Smith R, Wright IM. Measuring cystatin $\mathrm{C}$ to determine renal function in neonates. Pediatr Crit Care Med. 2013 Mar; $14(3): 318-22$.
9 Astor BC, Shafi T, Hoogeveen RC, Matsushita K, Ballantyne CM, Inker LA, et al. Novel markers of kidney function as predictors of ESRD, cardiovascular disease, and mortality in the general population. Am J Kidney Dis. 2012 May;59(5):653-62.

10 Zaleska-Kociecka M, Skrobisz A, Wojtkowska I, Grabowski M, Dabrowski M, Kusmierski $\mathrm{K}$, et al. Serum beta- 2 microglobulin levels for predicting acute kidney injury complicating aortic valve replacement. Interact Cardiovasc Thorac Surg. 2017 Oct;25(4):533-40.

11 Karlo J, Bhat BV, Koner BC, Adhisivam B. Evaluation of renal function in term babies with perinatal asphyxia. Indian J Pediatr. 2014 Mar;81(3):243-7. 
12 Haase M, Devarajan P, Haase-Fielitz A, Bellomo R, Cruz DN, Wagener G, et al. The outcome of neutrophil gelatinase-associated lipocalinpositive subclinical acute kidney injury: a multicenter pooled analysis of prospective studies. J Am Coll Cardiol. 2011 Apr;57(17):1752-61.

13 Askenazi DJ, Montesanti A, Hunley H, Koralkar R, Pawar P, Shuaib F, et al. Urine biomarkers predict acute kidney injury and mortality in very low birth weight infants. J Pediatr. 2011;159:907-12.e1.

14 Bellos I, Fitrou G, Daskalakis G, Perrea DN, Pergialiotis V. Neutrophil gelatinase-associated lipocalin as predictor of acute kidney injury in neonates with perinatal asphyxia: a systematic review and meta-analysis. Eur J Pediatr. 2018 Oct;177(10):1425-34.

15 Sweetman DU. Neonatal acute kidney injury - Severity and recovery prediction and the role of serum and urinary biomarkers. Early Hum Dev. 2017 Feb;105:57-61.

16 Filho LT, Grande AJ, Colonetti T, Della ÉS, da Rosa MI. Accuracy of neutrophil gelatinaseassociated lipocalin for acute kidney injury diagnosis in children: systematic review and meta-analysis. Pediatr Nephrol. 2017 Oct; 32(10):1979-88.

$17 \mathrm{Yu} \mathrm{H}$, Yanagisawa Y, Forbes MA, Cooper EH, Crockson RA, MacLennan IC. Alpha-1-microglobulin: an indicator protein for renal tubular function. J Clin Pathol. 1983 Mar;36(3): 253-9.
18 Treiber M, Gorenjak M, Pecovnik Balon B. Serum cystatin- $\mathrm{C}$ as a marker of acute kidney injury in the newborn after perinatal hypoxia/asphyxia. Ther Apher Dial. 2014 Feb;18(1):57-67.

19 Askenazi DJ, Ambalavanan N, Goldstein SL. Acute kidney injury in critically ill newborns: what do we know? What do we need to learn? Pediatr Nephrol. 2009 Feb;24(2):265-74.

20 Mehta RL, Kellum JA, Shah SV, Molitoris BA, Ronco C, Warnock DG, et al.; Acute Kidney Injury Network. Acute Kidney Injury Network: report of an initiative to improve outcomes in acute kidney injury. Crit Care. 2007; 11(2):R31.

21 Coca SG, Yalavarthy R, Concato J, Parikh CR Biomarkers for the diagnosis and risk stratification of acute kidney injury: a systematic review. Kidney Int. 2008 May;73(9):1008-16.

22 Askenazi DJ, Koralkar R, Hundley HE, Montesanti A, Parwar P, Sonjara S, et al. Urine biomarkers predict acute kidney injury in newborns. J Pediatr. 2012 Aug;161(2):270-5.e1.

23 Sarafidis K, Tsepkentzi E, Agakidou E, Diamanti E, Taparkou A, Soubasi V, et al. Serum and urine acute kidney injury biomarkers in asphyxiated neonates. Pediatr Nephrol. 2012 Sep;27(9):1575-82.

24 Nejat M, Pickering JW, Walker RJ, Westhuyzen J, Shaw GM, Frampton CM, et al. Urinary cystatin $\mathrm{C}$ is diagnostic of acute kidney injury and sepsis, and predicts mortality in the intensive care unit. Crit Care. 2010; 14(3):R85.
25 Herget-Rosenthal S, van Wijk JA, BröckerPreuss M, Bökenkamp A. Increased urinary cystatin $\mathrm{C}$ reflects structural and functional renal tubular impairment independent of glomerular filtration rate. Clin Biochem. 2007 Sep;40(13-14):946-51.

26 Hall IE, Koyner JL, Doshi MD, Marcus RJ, Parikh CR. Urine cystatin C as a biomarker of proximal tubular function immediately after kidney transplantation. Am J Nephrol. 2011; 33(5):407-13.

27 Yavuz S, Anarat A, Acartürk S, Dalay AC, Kesiktaş E, Yavuz M, et al. Neutrophil gelatinase associated lipocalin as an indicator of acute kidney injury and inflammation in burned children. Burns. 2014 Jun;40(4):64854.

28 Hoffman SB, Massaro AN, Soler-García AA, Perazzo S, Ray PE. A novel urinary biomarker profile to identify acute kidney injury (AKI) in critically ill neonates: a pilot study. Pediatr Nephrol. 2013 Nov;28(11): 2179-88.

29 Askenazi DJ, Koralkar R, Levitan EB, Goldstein SL, Devarajan P, Khandrika S, et al. Baseline values of candidate urine acute kidney injury biomarkers vary by gestational age in premature infants. Pediatr Res. 2011 Sep; $70(3): 302-6$. 